\title{
Characterization and density functional theory study of the antioxidant activity of quercetin and its sugar-containing analogues
}

\author{
Weirong Cai $\cdot$ Yong Chen $\cdot$ Liangliang Xie $\cdot$ \\ Hong Zhang $\cdot$ Chunyuan Hou
}

Received: 7 June 2013 / Revised: 24 August 2013 / Accepted: 27 August 2013 / Published online: 14 September 2013

(C) The Author(s) 2013. This article is published with open access at Springerlink.com

\begin{abstract}
Inhibition of free radicals using quercetin, hyperin and rutin is examined to determine their antioxidant effects and the structure-activity relationships of flavonoids. Two species of the free radicals are used, including hydroxyl radical $(\cdot \mathrm{OH})$ and superoxide anion radical $\left(\mathrm{O}_{2}^{-} \cdot\right)$. Density functional theory calculations under the level of B3LYP/6-311G (d) have been utilized to explore the structure, molecular properties and antioxidant abilities of the three flavonoids. Bond dissociation enthalpy (BDE) and frontier molecular orbital energy gap are investigated. They are compared with the experiment results assayed by the spectrophotometric. All of the flavonoids show a high activity on inhibiting $\cdot \mathrm{OH}$ and $\mathrm{O}_{2}^{-} \cdot$ radicals. Scavenging activity determined by half maximal inhibitory concentration $\left(\mathrm{IC}_{50}\right)$ values of the three flavonoids decreases in the order: quercetin $>$ hyperin $>$ rutin. The calculations show that quercetin owns the lowest BDE values, which agree well with the experimental results of antioxidant activity determined by $\mathrm{IC}_{50}$ values.
\end{abstract}

Keywords Flavonoids - Antioxidant activity - DFT . $\mathrm{BDE} \cdot \mathrm{IC}_{50}$

W. Cai $(\bowtie) \cdot$ Y. Chen $\cdot$ L. Xie $\cdot$ H. Zhang $\cdot$ C. Hou

College of Biological and Chemical Engineering,

Anhui Polytechnic University, Wuhu 241000,

People's Republic of China

e-mail: caiweirong0781@163.com

Y. Chen

e-mail: chengdscom@126.com

C. Hou

Dalian Institute of Chemical Physics, Chinese Academy

of Sciences, Dalian 116023, People's Republic of China

$\begin{array}{ll}\text { Abbreviations } \\ \text { DFT } & \text { Density functional theory } \\ \text { SARs } & \text { Structure-activity relationships } \\ \text { BDE } & \text { Bond dissociation enthalpy } \\ \text { IC }_{50} & \text { Half maximal inhibitory concentration } \\ \text { HOMO } & \text { Highest occupied molecular orbital } \\ \text { LUMO } & \text { Lowest unoccupied molecular orbital } \\ \text { IHBs } & \text { Intramolecular hydrogen bonds } \\ \text { ZPE } & \text { Zero-point energy } \\ \text { HAT } & \text { H-atom transfer } \\ \text { VC } & \text { Vitamin C } \\ \text { SA } & \text { Salicylic acid } \\ \cdot \mathrm{OH} & \text { Hydroxyl radical } \\ \mathrm{O}_{2}^{-} \cdot & \text { Superoxide anion radical }\end{array}$

\section{Introduction}

Free radicals such as hydroxyl radical (.OH) and superoxide anion radical $\left(\mathrm{O}_{2}^{-}\right.$.) are toxic compounds produced by the oxidative reaction. They have a high activity and can slowly cause severe damage to the cells [1]. They can attack the cell membrane or react with serum protease. Flavonoids typically show strong antioxidant activity by scavenging free radicals [2]. Some flavonoids' antioxidant capacity is equivalent with the antioxidants like vitamin $\mathrm{C}(\mathrm{VC})$ and vitamin $\mathrm{E}$ (VE) [3]. The radical scavenging properties of flavonoids are based on the phenol hydroxyl and the possibility of stabilization of the resulting phenoxyl radicals through hydrogen bonding or extended electron delocalization [4]. A number of studies have established the hierarchy of flavonoids in terms of their antioxidant activities and the corresponding structure-activity relationship (SAR) in vitro $[5,6]$. The existing data are principally focused on the effects of the position and number of hydroxyl group in 
molecule on antioxidant activity [7, 8], little is concern on the effect of sugar group in flavonoids. Meanwhile, the use of only one free radical is unsuitable for evaluating antioxidant capacity of flavonoids and the use of different methods in the various reaction systems is usually inconsistent. This contradiction hinders the understanding of the antioxidant capacity of flavonoids.

The aforementioned problem caused by the diversity of oxidants can be resolved through theoretical calculation. In theoretical calculations, several studies have explored and elucidated the antioxidant activity of flavonoids. Van Acker et al. [9] have used ab initio method to evaluate the antioxidant activity of flavonoids and found that the formation of intramolecular hydrogen bonds (IHBs) can be useful for the antioxidant activity. Figen et al. [10] have used AM1 to optimize the geometry of naringenin and genistein, while using ab initio RHF including full MP2 to calculate the electronic properties of the system. More recently, Monica et al. [11] have used density function theory (DFT) to study the antioxidant activity of apigenin, luteolin and taxifolin, and bond dissociation enthalpy (BDE) is used to measure flavonoids' antioxidant activity $[12,13]$. Their results show that B-ring of flavonoids is the most important site for antioxidant activity. So the study of the electronic and molecular properties is important to understand the mechanism of the antioxidant activity of these compounds.

This study is focused on the effect of sugar group in flavonoids. In our work, quercetin, hyperin (quercetin-3-galactoside) and rutin (quercetin-3-rhamnoglucoside) (Fig. 1) are chosen to investigate the SAR, especially the C-3 sugar group effect of flavonoids, and compared with the results

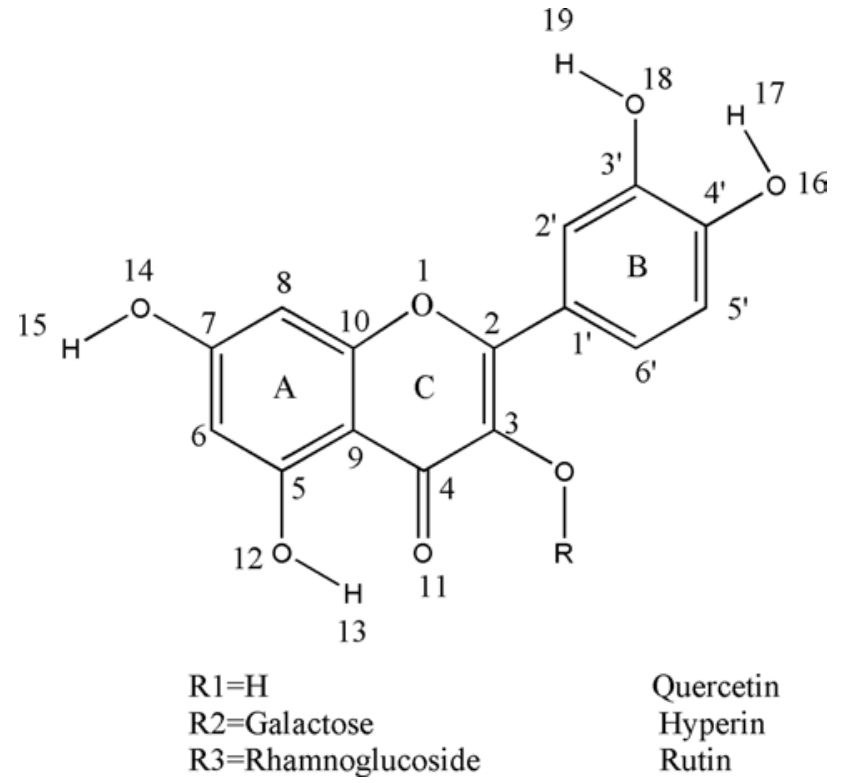

Fig. 1 Structures of quercetin, hyperin and rutin of two spectrophotometric assays. In this research, the geometrical features of the three compounds have been studied to confirm the capacity of their antioxidant activity, the $\mathrm{BDE}$ and other electronic properties together with highest occupied molecular orbital (HOMO) and lowest unoccupied molecular orbital (LUMO) energies have been elucidated. Experimental and theoretical values are compared with each other among the three flavonoids to discuss the effect of the sugar group in molecules. Property and capacity studies of flavonoids by experiment and theoretical calculation method can contribute to the ongoing interest in the understanding of flavonoids for better exploitation in the field of biology and food science.

\section{Materials and methods}

\section{Chemical reagents}

Quercetin, hyperin, rutin and VC are purchased from Aladdin Chemistry Co., Ltd. (Shanghai, China). Pyrogallol, Tris, hydrochloric acid, $\mathrm{FeSO}_{4}$, salicylic acid (SA), $\mathrm{H}_{2} \mathrm{O}_{2}$ and ethanol are purchased from Shanghai Chemical Reagent Co. Ltd. (Shanghai, China).

\section{.OH assay}

Fenton reaction method is used to create the $\cdot \mathrm{OH}$ radical generating system model in vitro. A slight modification is made according to Ref. [14]: $2 \mathrm{mmol} / \mathrm{L} \mathrm{FeSO}_{4} 5 \mathrm{~mL}$ and $6 \mathrm{mmol} / \mathrm{L} \mathrm{H}_{2} \mathrm{O}_{2} 5 \mathrm{~mL}$ are put into $25-\mathrm{mL}$ colorimetric tubes, the mixture is shaken well and then, $6 \mathrm{mmol} / \mathrm{L}$ salicylic acid is added to the mark. In a $37{ }^{\circ} \mathrm{C}$ water bath, keeping for $15 \mathrm{~min}$, the absorbance is measured at $510 \mathrm{~nm}$, which is recorded as $A_{0}$. Then, a certain concentration of $1 \mathrm{~mL}$ flavonoid solution is added to the reaction mixture. By keeping the water bath for $15 \mathrm{~min}$, its absorbance $A_{x}$ is measured. The antioxidant activities of the three flavonoids are compared with standard antioxidant VC.

Scavenging rate $(\%)=\left(A_{0}-A_{x}\right) / A_{0} \times 100$.

$\mathrm{O}_{2}^{-}$-assay

$\mathrm{O}_{2}^{-}$. radicals are produced by pyrogallol autoxidation method by making a slight modification according to Ref. [15]. About $0.1 \mathrm{~mL}$ of sample solution is added to $3.0 \mathrm{~mL}$ of $50 \mathrm{mM}$ tris- $\mathrm{HCl}$ buffer $(\mathrm{pH} 8.2)$ for $10 \mathrm{~min}$ at $25^{\circ} \mathrm{C}$, and then $0.1 \mathrm{~mL}$ of $3 \mathrm{mM}$ pyrogallol (positive) or $10 \mathrm{mM}$ $\mathrm{HCl}$ (control) are added in the assay system. After the mixture is rapidly shaken, its absorption value is determined at $325 \mathrm{~nm}$ in $0.5 \mathrm{~min}$ interval of $4 \mathrm{~min}$ (keeping the autooxidation rate of pyrogallol at $0.050-0.065 \mathrm{OD} / \mathrm{min})$. The 
antioxidant activities of the three flavonoids are compared with standard antioxidant VC.

Inhibition rate $(\%)=\left(\Delta A_{1} / \Delta t-\Delta A_{x} / \Delta t\right) /\left(\Delta A_{1} / \Delta t\right)$

$$
\times 100
$$

$\Delta A_{1} / \Delta t$ represents pyrogallol auto-oxidation reaction rate and $\Delta A_{x} / \Delta t$ means pyrogallol auto-oxidation reaction rate after adding the sample solutions.

Quantum chemical calculations

For ease of discussion, generic term FlaOH has been used to represent the antioxidant molecule. All computations have been implemented with the Gaussian 03 program package. DFT method under B3LYP/6-311G (d) basis set is adopted [16]. The structure optimization, frequency and energy calculations intend for phenoxyl radicals (FlaO-) for all the compounds are obtained by removing the $\mathrm{H}$-atom from the absolute minimum of neutral compounds $(\mathrm{FlaOH})$ at the same level of theory. While carrying out these calculations, unrestricted open shell (UB3LYP) method is employed. It has been observed from the harmonic vibration frequencies that all the conformations for these radicals have no imaginary frequency. The BDE is calculated by the following expressions: $\mathrm{BDE}=\mathrm{H}(\mathrm{FlaO} \cdot)+\mathrm{H}(\mathrm{H} \cdot)-\mathrm{H}(\mathrm{FlaOH})$ [17]. The zero-point energy (ZPE) is taken into account to correct the BDE values in the reaction at $298 \mathrm{~K}$.

\section{Statistical analysis}

All samples are prepared in triplicate. Each sample analysis is performed in triplicate. All results presented are mean \pm SD of at least three independent experiments. Statistical analysis (ANOVA with a statistical significance level set at $P<0.05$ and linear regression) is carried out with Minitab 15.

\section{Results and discussion}

Radical scavenging activity against $\cdot \mathrm{OH}$ radical

The scavenging rate of the three flavonoids and $\mathrm{VC}$ is shown in Fig. 2 and the half maximal inhibitory concentration $\left(\mathrm{IC}_{50}\right)$ values are shown in Table 1. From Fig. 2, we can know that the three flavonoids and VC have a high activity on scavenging $\cdot \mathrm{OH}$ radical in vitro and in the range of $0.01-0.08 \mathrm{mg} / \mathrm{mL}$; the clearance rate of the three flavonoids increases with the concentration increase. In the . $\mathrm{OH}$ assay, the highest scavenging activity against $\cdot \mathrm{OH}$ is quercetin while the lowest is rutin. The clearance rate of the three flavonoids does not differ much when the concentration is lower than $0.05 \mathrm{mg} / \mathrm{mL}$. When the concentration

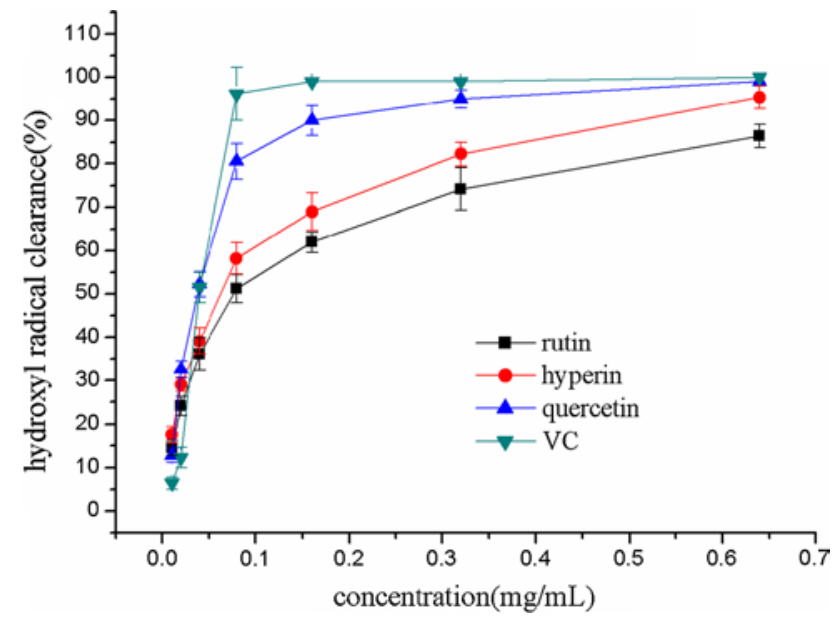

Fig. 2 Radical scavenging activity against $\cdot \mathrm{OH}$ radical

Table 1 Radical scavenging activities of rutin, hyperin, quercetin and VC

\begin{tabular}{llr}
\hline Compound & \multicolumn{1}{l}{$\mathrm{IC}_{50}(\mathrm{mg} / \mathrm{mL})$} & $\mathrm{O}_{2}{ }^{-} \cdot$ radical \\
\cline { 2 - 3 } & .OH radical & $0.42 \pm 0.09$ \\
\hline Quercetin & $0.034 \pm 0.005$ & $2.74 \pm 0.07$ \\
Hyperin & $0.059 \pm 0.004$ & $14.39 \pm 0.11$ \\
Rutin & $0.081 \pm 0.007$ & $0.26 \pm 0.08$ \\
Vitamin C & $0.034 \pm 0.004$ & \\
\hline
\end{tabular}

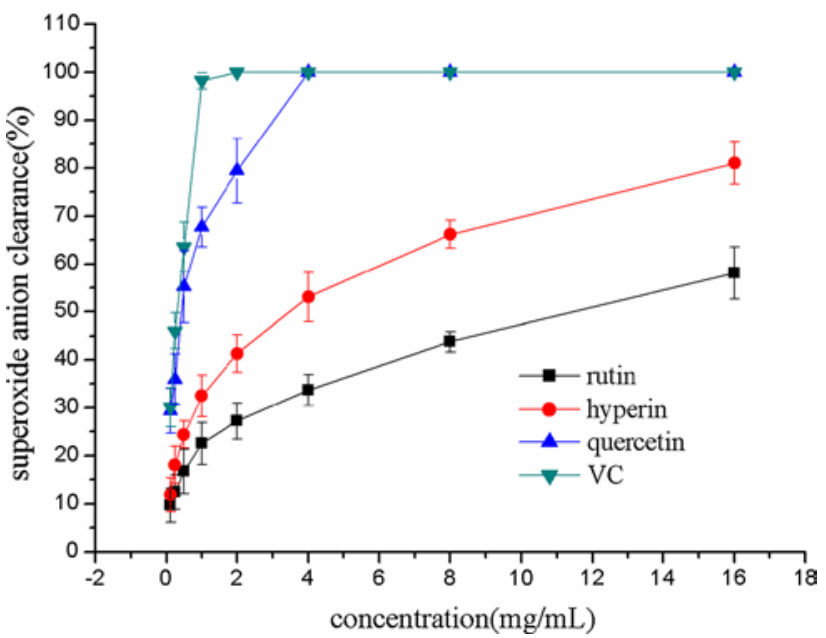

Fig. 3 Radical scavenging activity against $\mathrm{O}_{2}^{-}$. radical

of quercetin reaches $0.08 \mathrm{mg} / \mathrm{mL}$, its clearance rate can be $86.4 \%$ and its antioxidant activity is close to VC. The flavonoids exhibit different scavenging properties against $\cdot \mathrm{OH}$ radical depending upon the structure. $\mathrm{C} 3-\mathrm{OH}$ of flavonoids replaced by sugar group may inhibit its antioxidant and as the sugar group increases, antioxidant activity decreases. 
Fig. 4 The stable form of quercetin $\mathbf{a}$, hyperin $\mathbf{b}$ and rutin $\mathbf{c}$
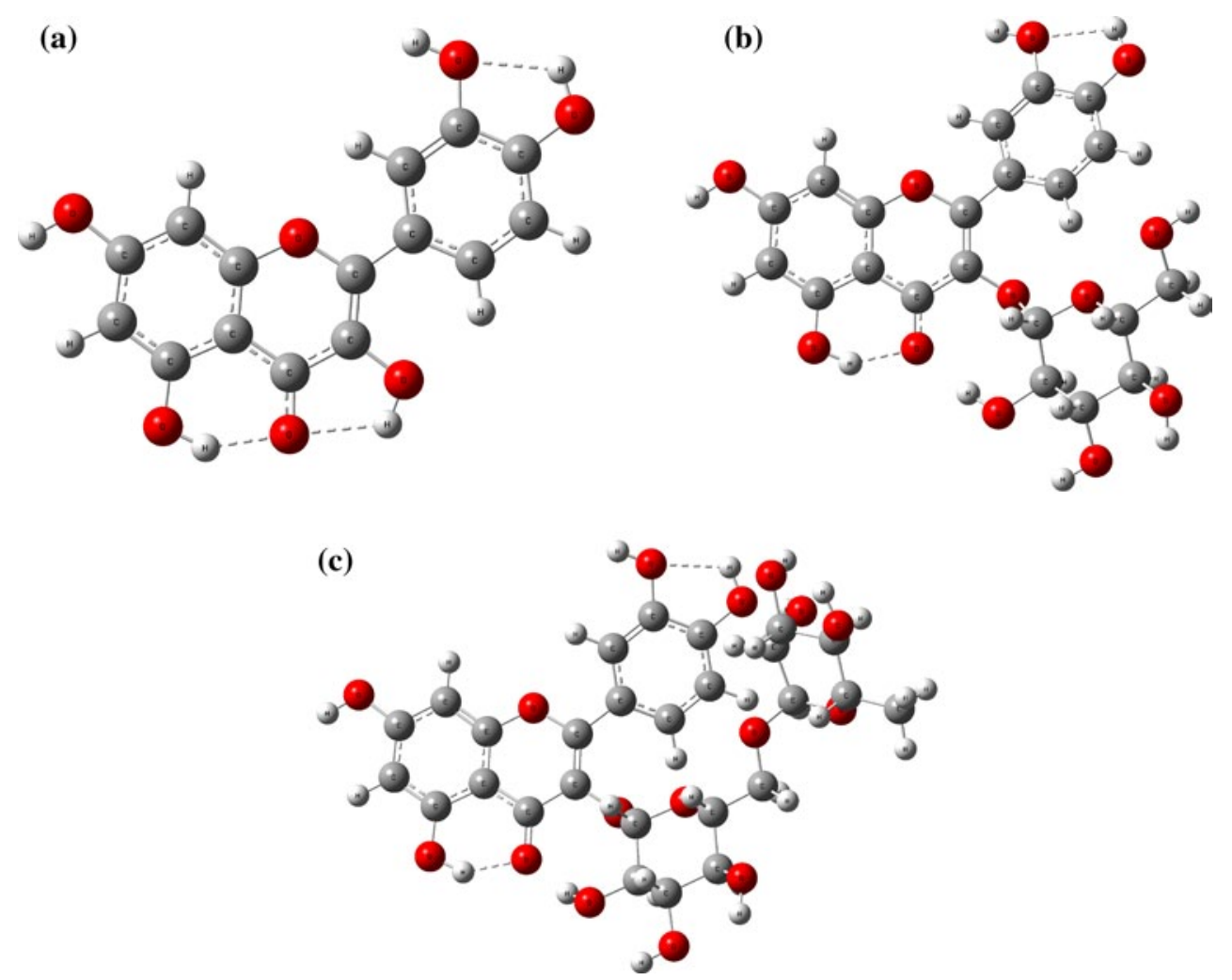

Sugar group may play a steric effect to inhibit antioxidant activity in the antioxidant process.

\section{Radical scavenging activity against $\mathrm{O}_{2}^{-} \cdot$ radical}

The scavenging activity of flavonoids and $\mathrm{VC}$ of the $\mathrm{O}_{2}{ }^{-}$. generated by the pyrogallol autoxidation system is examined. The $\mathrm{IC}_{50}$ values of quercetin, hyperin and rutin on scavenging $\mathrm{O}_{2}{ }^{-}$. radical are listed in Table 1 . The inhibition rate of pyrogallol autoxidation of the three flavonoids is displayed in Fig. 3. The scavenging activity of quercetin, hyperin and rutin decreases in the order: quercetin $>$ hyperin $>$ rutin. $\mathrm{IC}_{50}$ values of quercetin, hyperin, rutin and $\mathrm{VC}$ are $0.42,2.74,14.39$ and $0.26 \mathrm{mg} / \mathrm{mL}$, respectively. Quercetin shows the strongest antioxidant activity of the three flavonoids in different free radical generating system. The scavenging mechanism of $\mathrm{O}_{2}^{-}$. radical may be a coupled hydrogen atom electron transfer where the electron is transferred from the superoxide anion radical to flavonoid and the hydrogen atom is transferred from flavonoid to the radical [18]. Sugar group that substitutes $\mathrm{C} 3-\mathrm{OH}$ in flavonoids also plays a negative role in scavenging $\mathrm{O}_{2}^{-}$. radical.

\section{Geometry optimization}

Geometry optimization is carried out under the B3LYP/6$311 \mathrm{G}$ (d) basis set of Gaussian 03. The optimized structures
Table 2 The bond distances, bond angles and dihedral angles of the three flavonoids

\begin{tabular}{lrrr}
\hline Geometry & \multicolumn{1}{l}{ Quercetin } & \multicolumn{1}{l}{ Hyperin } & \multicolumn{1}{l}{ Rutin } \\
\hline Bond distances $(\AA)$ & & & \\
$R(12,13)$ & 0.99515 & 0.99638 & 0.99575 \\
$R(14,15)$ & 0.97194 & 0.97198 & 0.97205 \\
$R(16,17)$ & 0.97491 & 0.97591 & 0.97710 \\
$R(18,19)$ & 0.96989 & 0.96958 & 0.96956 \\
Bond angles $\left(^{\circ}\right)$ & & & \\
$A(5,12,13)$ & 109.76169 & 109.22203 & 109.47016 \\
$A\left(1,2,1^{\prime}\right)$ & 112.45586 & 110.47392 & 111.12312 \\
Dihedral angles $\left(^{\circ}\right)$ & & & \\
$D\left(3,2,1^{\prime}, 2^{\prime}\right)$ & -179.99758 & 154.82533 & -162.03076 \\
\hline
\end{tabular}

of the most stable conformation of quercetin, hyperin and rutin along with the pattern of IHBs are shown in Fig. 4. A part of calculating bond distances, bond angles and dihedral angles is displayed in Table 2. Vibration frequencies which calculated in the same level of theory have confirmed stationary points with no negative eigenvalues observed in the force constant matrix (Fig. 4).

From the optimized molecular structure of the three flavonoids, it can be seen that the 2,3-double bond exists in the three flavonoids, and it ensures $\pi$-electron delocalization between the $\mathrm{B}$ - and $\mathrm{C}$-rings, which contribute to the 
stabilization of $\mathrm{FlaO}$, after H-abstraction [19]. For quercetin, 2, 3-double bond and the $\mathrm{C} 3-\mathrm{OH}$ group make it planar and $\mathrm{C}$-ring expands the planar structure as the connect ring. It is beneficial for the $\mathrm{H}$-atom transfer (HAT) and it forms the more stable half-quinone free radical in scavenging the free radical. Compared with all phenol hydroxyl groups on quercetin molecular, $\mathrm{C5}-\mathrm{OH}$ bond length is noticeably longer than the others. It may be related to the ketone group in $\mathrm{C}$-ring which forms an IHB with the $\mathrm{C} 5-\mathrm{OH}$ group, so that the $\mathrm{C} 5-\mathrm{OH}$ bond distances are stretched. The hydrogen bond is also found between the $3^{\prime}-\mathrm{OH}$ and $4^{\prime}-\mathrm{OH}$ groups in the three flavonoid compounds by comparing the bond distance as shown in Table 2. The two hydrogen bonds, between the $3^{\prime}-\mathrm{OH}$ and $4^{\prime}-\mathrm{OH}$ groups, the 5-OH and $\mathrm{C} 4-\mathrm{O}$ ketone groups may use for stabilizing the structure [20].

In contrast, the benzopyrone and phenyl rings are not planar in rutin and hyperin. The calculated A-to-B dihedral angles of rutin and hyperin are $17.97^{\circ}$ and $25.18^{\circ}$, respectively. The A-to-B dihedral angles may be caused by the sugar group in the $\mathrm{C}$-ring. Different $\mathrm{A}$-to-B dihedral angles lead the flavonoids molecule conformation to reverse and make molecule lose coplanar varying degrees. It will weaken the electronic distribution of flavonoid molecules, and be an important factor of different antioxidant activity in unique flavonoid compounds.

\section{BDE values}

It is appropriately certificated that $\mathrm{BDE}$ for the $\mathrm{O}-\mathrm{H}$ bond is of specific importance to acquaint the mechanism of radical scavenging activity [21], because a weak $\mathrm{O}-\mathrm{H}$ bond means the faster reaction rate and high antioxidant activity. Free radical scavenging capacity of flavonoids is also generally related to the existence of $\mathrm{OH}$ groups in a specific position on the flavonoid core.

Optimization of the geometry of the radical species formed after HAT of the three flavonoid compounds is carried out starting from the optimized geometry of the absolute minimum structures of the neutral version. The removal of $\mathrm{H}$-atom from the $\mathrm{C}^{\prime}, \mathrm{C}^{\prime}, \mathrm{C} 5$ and $\mathrm{C} 7$ positions in the three flavonoid compounds gives rise to four radical species. The radical species created by the removal of $\mathrm{H}$-atom from the $\mathrm{C}^{\prime}-\mathrm{OH}$ group of quercetin is called as $\mathrm{C}^{\prime}-\mathrm{OH}$ quercetin radical species. In the same way, remaining three radical species are generated and named. The same methodology has been adopted for the generation and naming of the four radical species of rutin and hyperin. Geometry optimizations on the radicals are calculated by the UB3LYP/6-311G (d) method in gas phase. During the optimization of the radical species of the three flavonoid compounds, no geometrical parameter constraint is employed [22].
Table 3 The computed BDE values for the three flavonoid compounds $(\mathrm{kcal} / \mathrm{mol})$

\begin{tabular}{lllll}
\hline Specials & $5-\mathrm{OH}$ & $7-\mathrm{OH}$ & $3^{\prime}-\mathrm{OH}$ & $4^{\prime}-\mathrm{OH}$ \\
\hline Quercetin & 89.7 & 76.3 & 65.8 & 75.9 \\
Hyperin & 90.5 & 77.4 & 67.6 & 76.2 \\
Rutin & 94.8 & 82.5 & 73.8 & 81.1 \\
\hline
\end{tabular}

The computed BDE values for the three flavonoid compounds are shown in Table 3 and from it we can see that quercetin has the lowest $\mathrm{BDE}$ in $3^{\prime}-\mathrm{OH}$ at $65.8 \mathrm{kcal} /$ mol; $3^{\prime}-\mathrm{OH}$ in rutin and hyperin also provides a low BDE value at 73.8 and $67.6 \mathrm{kcal} / \mathrm{mol}$, respectively. In our calculation, BDE values in the gas phase of the four radical species of quercetin, rutin and hyperin leading to the consistent following BDE sequence: $\mathrm{C}^{\prime}-\mathrm{OH}<\mathrm{C}^{\prime}{ }^{\prime}-$ $\mathrm{OH}<\mathrm{C} 7-\mathrm{OH}<\mathrm{C} 5-\mathrm{OH}$. It clearly confirms that H-transfer from B-ring $\left(3^{\prime}-\mathrm{OH}\right)$ is much easier than from A-ring (5$\mathrm{OH}$ and $7-\mathrm{OH}$ ), and $\mathrm{BDE}$ of $\mathrm{C} 5-\mathrm{OH}$ group is higher than others. This difference is related to the fact that a hydrogen bond exists between the $\mathrm{C} 5-\mathrm{OH}$ group and adjacent ketone group. The result is that BDE on this site is higher because the $\mathrm{H}$-atom removal implies the cleavage of the hydrogen bond. Similarly, H-atom removal from $\mathrm{C}^{\prime}-\mathrm{OH}$ also implies the breaking of the hydrogen bond. B-ring is more active than A-ring. The BDE value of $\mathrm{C}^{\prime}-\mathrm{OH}$ in B-ring is lower than the $\mathrm{OH}$ in A-ring though the hydrogen bond exists between $\mathrm{C}^{\prime}{ }^{\prime}-\mathrm{OH}$ and $\mathrm{C}^{\prime}-\mathrm{OH}$. These findings are consistent with the results which are found by Dhaouadi et al. [23, 24]. Judging from the lowest BDE, the number of phenol hydroxyl group for each natural flavonoids and its relative energy values are obtained according to the HAT mechanism; our results are in good agreement with those obtained in the experiment [25-27], and we can describe the activities of the flavonoids under study in the following order: quercetin $>$ hyperin $>$ rutin, which are in accordance with the experimental results determined by spectrophotometric assays.

Frontier molecular orbital theory

The frontier orbital calculated for the three flavonoid compounds at the level of B3LYP/6-311G (d) in the gas phase is shown in Fig. 5, and the frontier orbital energy is also indicated in Table 4. The active site can be demonstrated visually by the distribution of frontier orbital. From Fig. 5, we can find that the HOMO of the three flavonoid compounds is mainly distributed in the B-ring, while the LUMO allocated in the C-ring. So the higher ability to respond to the functional site is mainly focused on B-ring and the conjugate part. The electron-donating capability of a molecule can be determined by the values of HOMO; a 
Fig. 5 The frontier orbital calculated for quercetin, hyperin and rutin

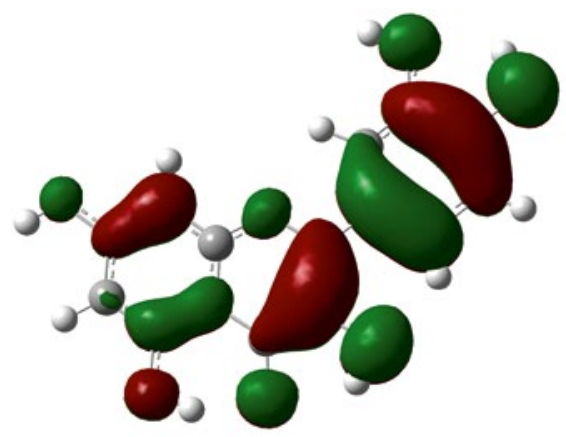

quercetin (HOMO)

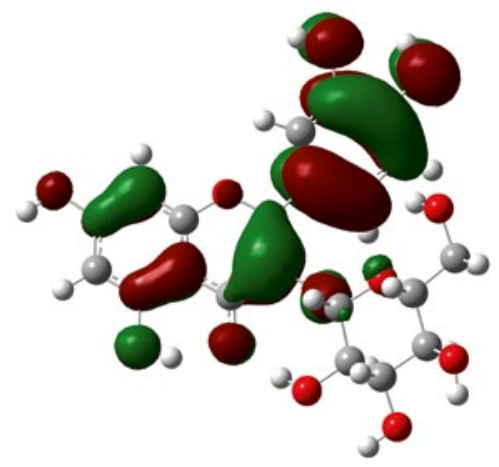

hyperin (HOMO)

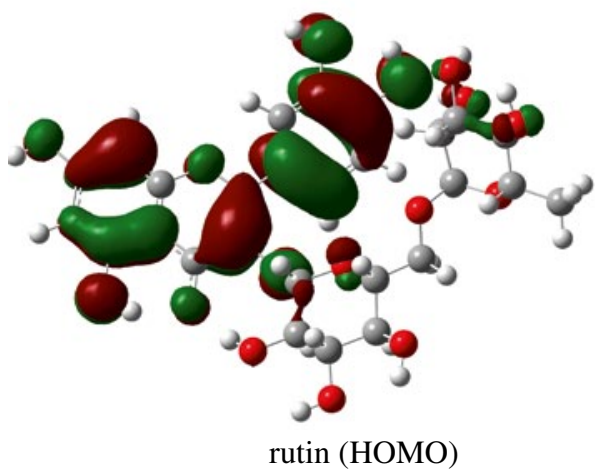

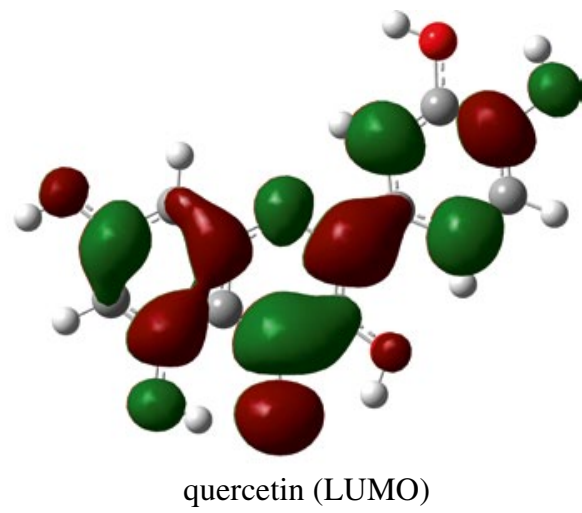

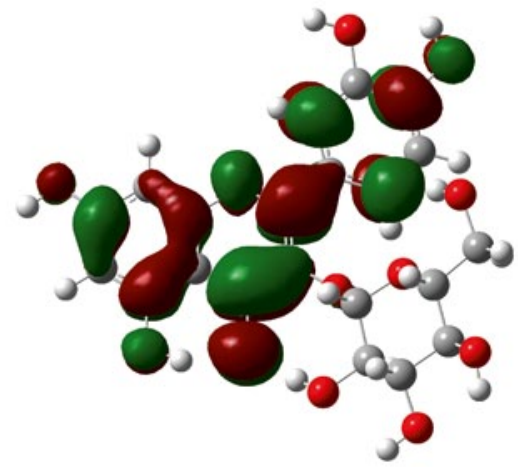

hyperin (LUMO)

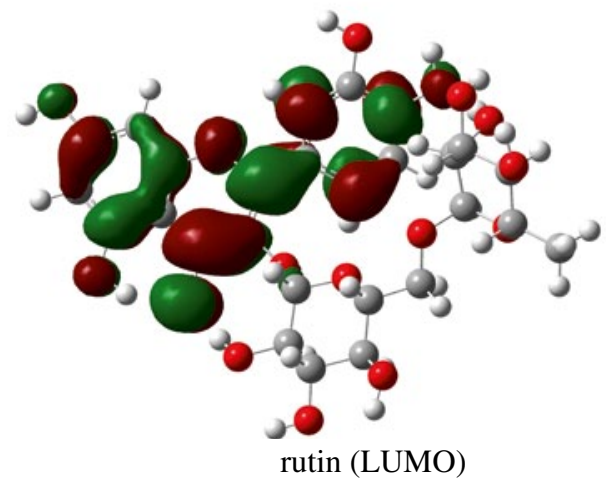

Table 4 The frontier orbital energy and energy gap of quercetin, hyperin and rutin $(\mathrm{eV})$

\begin{tabular}{llll}
\hline Flavonoids & HOMO & LUMO & $E_{\text {gap }}$ \\
\hline Quercetin & -5.93 & -2.37 & 3.56 \\
Hyperin & -6.18 & -2.57 & 3.61 \\
Rutin & -6.67 & -2.93 & 3.74 \\
\hline
\end{tabular}

high HOMO corresponds with a strong capability to donate electrons [28]. It can be seen from Table 4 that quercetin possesses the highest HOMO among the three flavonoid compounds, while rutin owns the lowest. Based on our calculations, the trend for computing HOMO is scarcely different from that of BDE, correspond to the arrangement: quercetin $>$ hyperin $>$ rutin.

Another parameter to be given importance is the energy gap $\left(E_{\text {gap }}\right)$ of these three compounds. The band gap of quercetin flavonoid compound is calculated as $3.56 \mathrm{eV}$, whereas for rutin and hyperin it is 3.61 and $3.74 \mathrm{eV}$, respectively. The band gap energy difference between quercetin and hyperin is only $0.05 \mathrm{eV}$, and the difference in the band gap energies of quercetin and rutin is found to be $0.18 \mathrm{eV}$. The lower the $E_{\text {gap }}$, the easier the electrons inspire and the more vigorous the antioxidant. The low magnitude of the band gap energy indicates that the flavonoid compounds could be a highly reactive system. The different energy of $E_{\text {gap }}$ in flavonoid compounds 
is a representation of molecular planarity and a quantum electronic feature of the frontier orbital [29]. The analysis of band gap values obtained for quercetin, hyperin and rutin are in accord with the results of the frontier orbital theory.

\section{Conclusion}

In this paper, we have utilized two spectrophotometric assays and B3LYP/6-311G (d) method to explore the structural features and molecular properties of three naturally occurring flavonoid compounds including quercetin, rutin and hyperin. The study has concerned the determination of scavenging radical activity in vitro and geometrical properties in order to identify the contribution of the sugar group of the antioxidant activity. In addition to that various molecular descriptors such as the BDE, HOMO and $E_{\text {gap }}$ of the flavonoid compounds have also been obtained and studied, which are relevant to show evidence of antioxidant activity.

Our study indicates that the dihedral angles between A-ring and B-ring of rutin and hyperin may be caused by the sugar group at C3. It may weaken the antioxidant activity and it is an important factor of different antioxidant activity in different flavonoid compounds. The role of B-ring is found to dominate the antioxidant property of the three compounds based on the analysis of the results. Comparison among the three considered molecules indicates quercetin as the flavonoid that requires the lowest energy for $\mathrm{H}$-atom transfer mechanisms. Low values of the BDE and energy gap show that the ortho-phenolic hydroxyl group on the B-ring is the most active. The activities of the three flavonoid compounds may be arranged in the following order: quercetin $>$ hyperin $>$ rutin and it is an agreement with the experiment results we get.

The above finding undoubtedly indicates that the sugar group in the molecule plays a negative effect for antifree radical activity of flavonoids. C-ring 3-OH replaced by sugar group can decrease the antioxidant activity of flavonoids. By increasing the number of sugar group, the antifree radical effects decrease sequentially. We hope the study of the property and capacity of the antioxidant can contribute to the ongoing interest in the understanding of flavonoids for better exploitation in the field of biology and food science.

Acknowledgments This work is financed by National Nature Science Foundation of China (No. 31171753), Open Project of Key Laboratory of Carbohydrate Chemistry \& Biotechnology Ministry of Education (No. 312 KLCCB-KF201202), and International Science and Technology Cooperation Program of Anhui Province (No. 1008703035).
Conflict of interest None.

Compliance with Ethics Requirements This article does not contain any studies with human or animal subjects.

Open Access This article is distributed under the terms of the Creative Commons Attribution License which permits any use, distribution, and reproduction in any medium, provided the original author(s) and the source are credited.

\section{References}

1. Valko M, Leibfritz D, Moncol J, Cronin MT, Mazur M, Telser J (2007) Free radicals and antioxidants in normal physiological functions and human disease. Int J Biochem Cell B 39(1):44-84

2. Claudine M, Augustin S, Christine M, Christian R, Liliana J (2004) Polyphenols: food sources and bioavailability. Am J Clin Nutr 79:727-747

3. Wolf B, Werner H, Christa M, Manfred S (1990) Flavonoids as antioxidants: determination of radical-scavenging efficiencies. Method Enzymol 186:343-355

4. Nessa F, Ismail Z, Mohamed N, Haris MRHM (2004) Free radical-scavenging activity of organic extracts and of pure flavonoids of Blumea balsamifera DC leaves. Food Chem 88(2):243-252

5. Masuoka N, Matsuda M, Kubo I (2012) Characterisation of the antioxidant activity of flavonoids. Food Chem 131(2):541-545

6. Takasawa R, Takahashi S, Saeki K, Sunaga S, Yoshimori A, Tanuma S (2008) Structure-activity relationship of human GLO I inhibitory natural flavonoids and their growth inhibitory effects. Bioorg Med Chem 16(7):3969-3975

7. Trouillas P, Marsal P, Siri D, Lazzaroni R, Duroux J-L (2006) A DFT study of the reactivity of $\mathrm{OH}$ groups in quercetin and taxifolin antioxidants: the specificity of the $3-\mathrm{OH}$ site. Food Chem 97(4):679-688

8. Zhang D, Chu L, Liu Y, Wang A, Ji B, Wu W, Zhou F, Wei Y, Cheng Q, Cai S, Xie L, Jia G (2011) Analysis of the antioxidant capacities of flavonoids under different spectrophotometric assays using cyclic voltammetry and density functional theory. $\mathrm{J}$ Agric Food Chem 59(18):10277-10285

9. Acker SABE, Groot MJ, Berg DJ, Tromp MNJL, Kelder GDO, Vijgh WJF, Bast A (1996) A quantum chemical explanation of the antioxidant activity of flavonoids. Chem Res Toxicol 9:1305-1312

10. Figen E, Sakir E (2002) Theoretical investigation of flavonoids naringenin and genistein. J Mol Struct Theochem 583:163-167

11. Monica L, Immaculada PP, Nino R, Marirosa T (2004) Structure, conformation, and electronic properties of apigenin, luteolin, and taxifolin antioxidants. A first principle theoretical study. J Phys Chem A 108:92-96

12. Trouillas $P$, Fagnère $C$, Lazzaroni R, Calliste $C$, Marfak $A$, Duroux J-L (2004) A theoretical study of the conformational behavior and electronic structure of taxifolin correlated with the free radical-scavenging activity. Food Chem 88(4):571-582

13. Zhang H-Y, Ji H-F (2006) How vitamin E scavenges DPPH radicals in polar protic media. New J Chem 30:503-504

14. Caillet S, Yu H, Lessard S, Lamoureux G, Ajdukovic D, Lacroix $M$ (2007) Fenton reaction applied for screening natural antioxidants. Food Chem 100(2):542-552

15. Li J, Zhang M, Zheng T (2009) The in vitro antioxidant activity of lotus germ oil from supercritical fluid carbon dioxide extraction. Food Chem 115(3):939-944

16. Senthil kumar K, Kumaresan R (2012) A DFT study on the structural, electronic properties and radical scavenging mechanisms 
of calycosin, glycitein, pratensein and prunetin. Comput Theor Chem 985:14-22

17. Christopher JP, Paul MM, Leo R (1999) An assessment of theoretical procedures for the calculation of reliable radical stabilization energies. J Chem Soc Perkin Trans 2:2305-2313

18. Ghiasi M, Heravi MM (2011) Quantum mechanical study of antioxidative ability and antioxidative mechanism of rutin (vitamin P) in solution. Carbohydr Res 346(6):739-744

19. Zhang J, Du F, Peng B, Lu R, Gao H, Zhou Z (2010) Structure, electronic properties, and radical scavenging mechanisms of daidzein, genistein, formononetin, and biochanin A: a density functional study. J Mol Struct Theochem 955(1-3):1-6

20. Cornard JP, Boudet AC, Merlin JC (1999) Theoretical investigation of the molecular structure of the isoquercitrin molecule. $\mathrm{J}$ Mol Struct 508:37-49

21. Sadasivam K, Kumaresan R (2011) Antioxidant behavior of mearnsetin and myricetin flavonoid compounds-a DFT study. Spectrochim Acta A 79(1):282-293

22. Zhang H-Y (1998) Selection of theoretical parameter characterizing scavenging activity of antioxidants on free radicals. J Am Oil Chem Soc 75:1705-1709

23. Dhaouadi Z, Nsangou M, Garrab N, Anouar EH, Marakchi K, Lahmar S (2009) DFT study of the reaction of quercetin with and radicals. J Mol Struct Theochem 904(1-3):35-42
24. Vagánek A, Rimarčík J, Lukeš V, Klein E (2012) On the energetics of homolytic and heterolytic $\mathrm{OH}$ bond cleavage in flavonoids. Comput Theor Chem 991:192-200

25. Seyoum A, Asres K, El-Fiky FK (2006) Structure-radical scavenging activity relationships of flavonoids. Phytochemistry 67(18):2058-2070

26. Jin Y, Lu Y, Han G-Z, Li W-P, Zhou Q, Jin F-X, Yu H-S (2007) Structure-effect study of quercetin and its sugar-containing analogues as antioxidants in in vitro $\mathrm{H}_{2} \mathrm{O}_{2}$-induced cell injury model. Asian J Pharmacokinet Phar 7(4):295-301

27. Astrid G, Elizabeth J, Chris FH (1997) Comparison of the antioxidant activity of aspalathin with that of other plant phenols of rooibos tea (Aspalathus linearis), $\alpha$-tocopherol, BHT, and BHA. J Agric Food Chem 45:632-638

28. Clare BW (1995) Charge transfer complexes and frontier orbital energies in QSAR: a congeneric series of electron acceptors. J Mol Struct Theochem 337:139-150

29. Hatch FT, Lightstone FC, Colvin ME (2000) Quantitative structure-activity relationship of flavonoids for inhibition of heterocyclic amine mutagenicity. Environ Mol Mutagen 35:279-299 